\title{
Objectivity in the classification of tumours of the nasal epithelium
}

\author{
L. Michaels \\ M.D., F.R.C.Path., F.R.C.P. (C.) \\ V. J. HYAMS \\ M.D., M.C. \\ Department of Pathology, Institute of Laryngology and Otology, London and \\ Otolaryngic Section, Armed Forces Institute of Pathology, Washington D.C., U.S.A.
}

\section{Summary}

A survey of tumours derived from each of the four cell types of nasal epithelium is presented. Criticism is levelled at the adoption of additional terms for tissue types such as lympho-epithelium and transitional cell epithelium and tumours said to be derived from them. Electron microscopy is of assistance in classification particularly in the detection of evidence of keratin synthesis. The proposed classification of tumours of the nasal epithelium is:

(1) Pseudostratified columnar epithelium: (a) papillary adenoma, (b) papillary carcinoma.

(2) Squamous epithelium: (a) everted squamous papilloma, (b) inverted papilloma, (c) squamous carcinoma of any grade of differentiation from well differentiated to undifferentiated.

(3) Melanocyte: malignant melanoma.

(4) Olfactory neuroepithelium: olfactory neuroblastoma.

In the process of classification of tumours the pathologist apportions each tumour according to the normal tissue growth pattern which it most closely resembles. The method of classification is histological using the light microscope in most cases; in a few cases the electron microscope gives further information as to tissue of origin.

The purposes of such histological classification are twofold.

(1) To help in assessing the behaviour of the tumour in the patient. The addition of the terms benign or malignant gives some indication of the expected behaviour, although it is admitted that these terms are vague and inaccurate and that each tumour type requires a more specific description of behaviour. Such a description is based on a study of the behaviour of tumours with similar histological appearances in previous cases over a prolonged period of time, and their response to treatment particularly surgery and irradiation.

(2) To lead to further scientific information regarding the tumour, notably factors in its causation.

Correspondence: Professor L. Michaels, Department of Pathology, Institute of Laryngology and Otology, 330 Gray's Inn Road, London WC1X 8EE.
To attain these aims the histological assessment of the tumour must be objectively based. Such objectivity has at times been lacking in the classification of tumours of the nasal passages. The purpose of this paper is to describe an objective classification of such tumours based on our special experience with these specimens. We further discuss reasons for the loss of objectivity in the literature on them. Since the problem has been mainly concerning tumours of the nasal epithelium discussion will be confined to such tumours; neoplasms of seromucinous glands and deeper structures will not be mentioned.

Materials and methods

Light microscopical histological study was carried out on a wide range of nasal tumours at the Institute of Laryngology and Otology, London, England, and the ENT section of the Armed Forces Institute of Pathology, Washington, D.C. In each case paraffin sections were stained with $\mathrm{HE}$ and additional staining methods were used as required.

In certain cases electron microscopical examination of selected areas of the tumour was carried out. For this purpose fragments of the tumour were fixed in $3 \%$ glutaraldehyde solution in Sörensen's $0.1 \mathrm{M}$ phosphate buffer at 7.3 for $4 \mathrm{hr}$, rinsed in $0.1 \mathrm{M}$ Sörensen's buffered solution and further fixed in osmium tetroxide and veronal acetate for $1 \mathrm{hr}$. The specimens were then embedded in Araldite and sections were cut on an LKB ultramicrotome and stained with lead citrate and uranyl acetate solutions.

\section{Results and conclusions}

It is first necessary to define the types of cell which may be found in normal nasal epithelium. Each cell type would be expected to produce its own tumour forms and classification should be based on this.

\section{The cell types of the nasal epithelium}

There are four cell types in the normal nasal epithelium:

(1) Pseudostratified columnar epithelium. This is the basic cellular pattern of the epithelium of the nose. It should be noted that the surface epithelial 


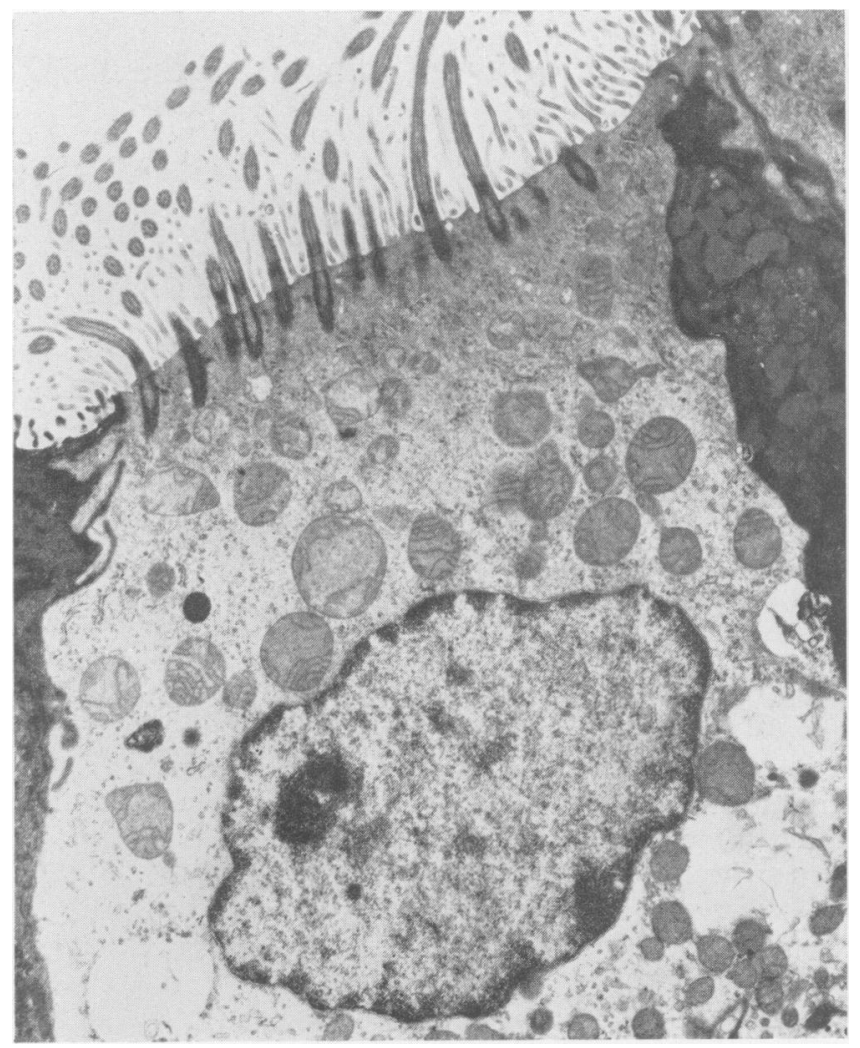

Fig. 1. Normal pseudostratified columnar epithelium from the trachea of a mouse. Note two types of cell: a ciliated cell occupying most of the figure shows numerous ciliary processes emerging from the upper surface. To right of it is a darkly stained goblet cell with cytoplasm almost completely occupied by secretory granules. Electron micrograph $\times 7200$.

cells may be either ciliated or become distended with mucus (Fig. 1).

(2) Squamous epithelium. This is not found in the infantile nose at birth. Later in life patches of squamous epithelium develop in the nasopharynx; such metaplastic foci are unusual in the nasal cavity and nasal sinuses except in the presence of a source or irritation, e.g. nasal polyps. A squamous epithelium (more accurately, stratified squamous epithelium) shows horizontal alignment of its cells, unlike the vertically aligned pseudostratified epithelium. In the latter all cells send processes to the basement while in the squamous epithelium only the basal layer of cells have contact with the basement membrane. The electron microscopical appearances of squamous epithelium are characteristic with increasing numbers of tonofibrils towards the surface, increasing keratinization and increasing numbers of desmosomes between the cells in the same direction and the presence of keratohyaline granules in cells about to become completely keratinized (Figs 2 and 3 ). Frequently the basal 'reserve' cells of pseudo-3. stratified columnar epithelium contain tonofibrils ando it is from such cells that the squamous epithelium. probably arises in the process of squamous metaplasia. Nevertheless the fundamental difference between pseudostratified epithelium and squamous epithelium is the existence of processes of keratin synthesis in all cells of the latter.

Sometimes the hallmarks of keratin synthesis may be absent by the light microscope in an epithelium which appears to have undergone some stratification $\mathcal{O}^{\circ}$ (Fig. 4). These specific appearances are, particularly $N_{\text {N }}^{N}$ keratinization and prickle cell formation. Gould, Wenk and Sommers (1971) showed that irritation byo the presence of a stainless steel pellet produced stratification and loss of cilia and mucous globules? in a pseudostratified epithelium experimentally, buto not keratinization by electron microscopical criteria우 To produce keratinization it was necessary to coato 


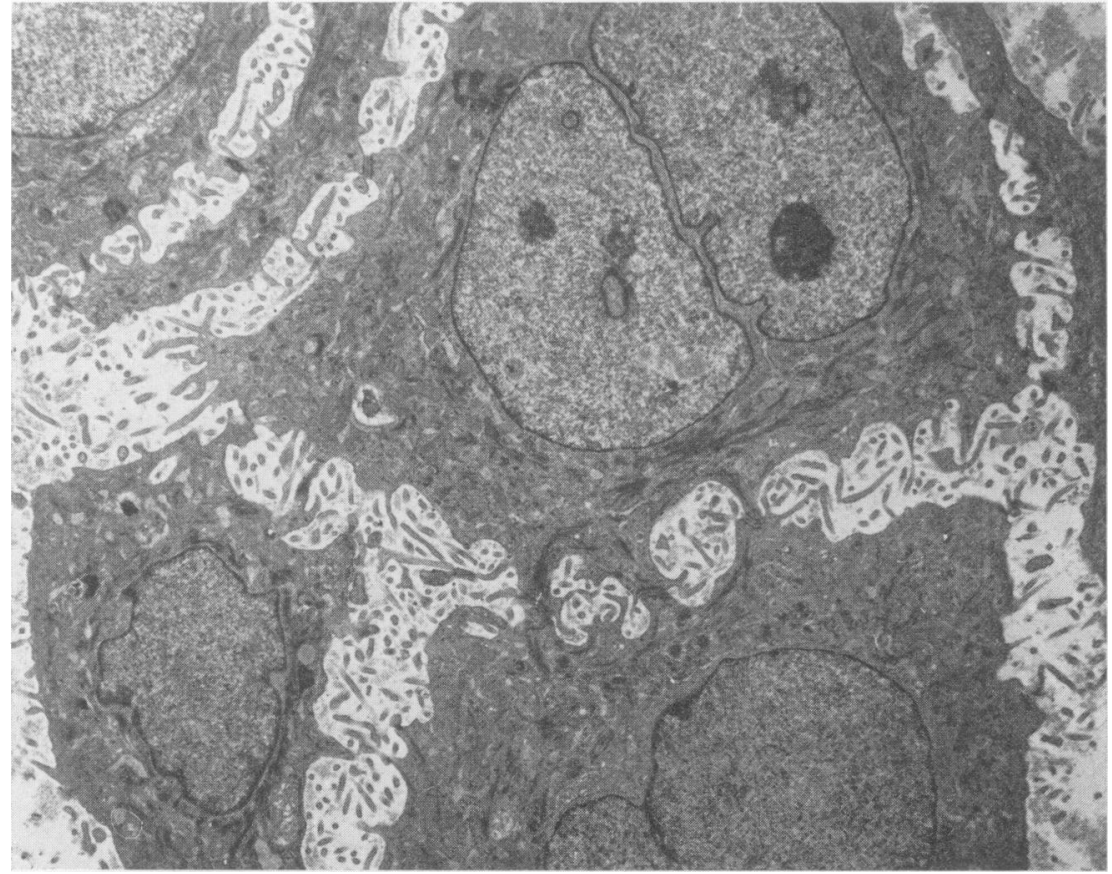

Fig. 2. Differentiated squamous cells from a squamous carcinoma. Clumps of fibrils are present in the cytoplasm of each cell. The cells are joined by microvilli on some of which desmosomes can be identified. Electron micrograph $\times 3600$.

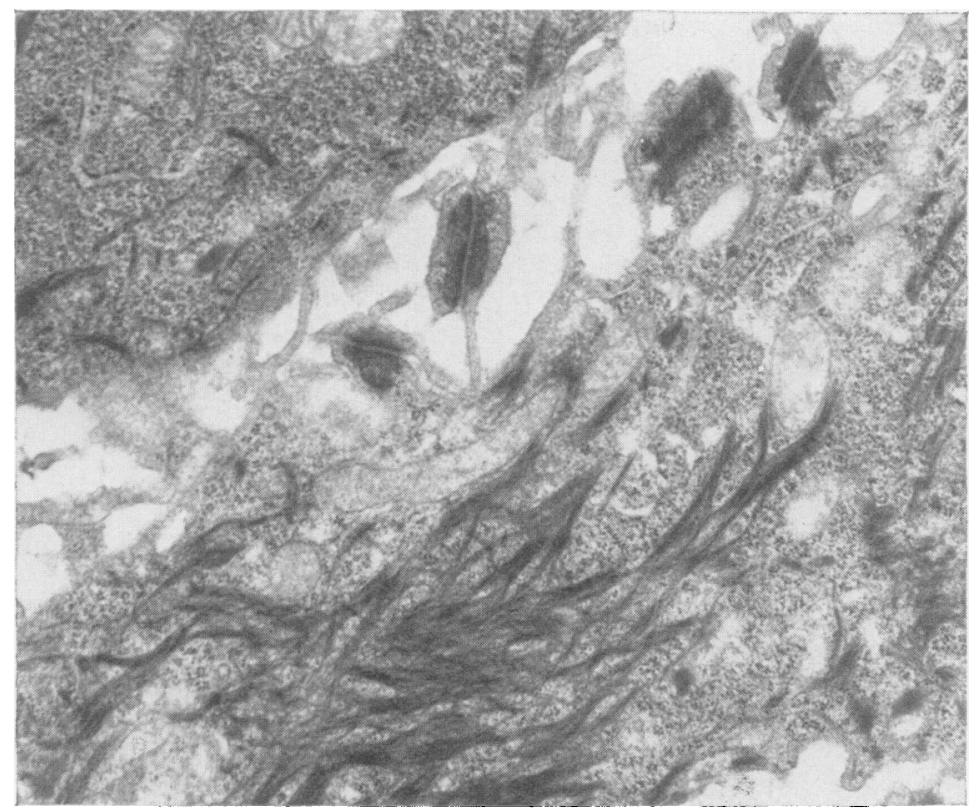

Fig. 3. Two squamous carcinoma cells. The abundant tonofibrils and desmosomes from the region of which they emanate constitute evidence for keratin synthesis in these cells. Fine cytoplasmic granules, probably glycogen are also abundant; this substance also frequently is present in differentiated squamous cells. Electron micrograph $\times 6800$. 


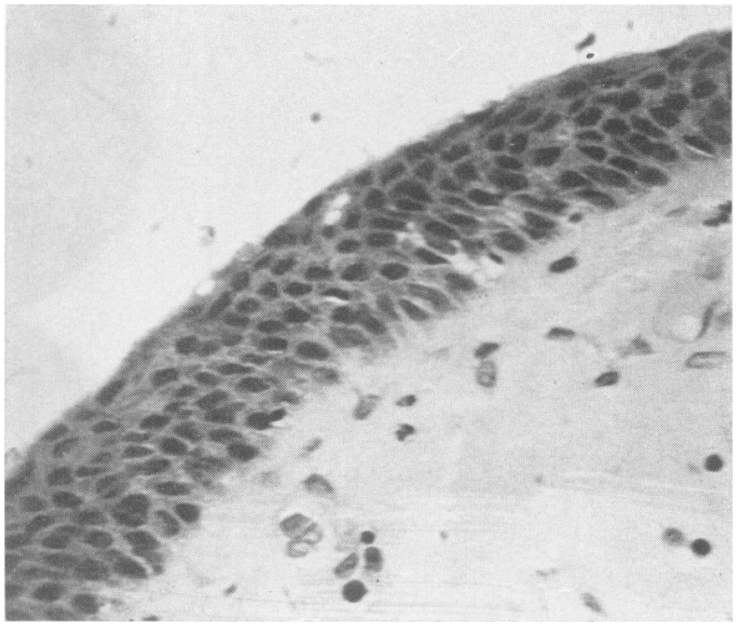

Fig. 4. So-called 'transitional' epithelium from surface of nasal polyp. Pseudostratified columnar epithelium is replaced by stratified epithelium, but features of keratinization are not present. HE $\times 360$.

the pellets with 3-methylcholanthrene-a carcinogenic agent. The non-keratinizing but stratified epithelium found in the respiratory tract has been described as 'transitional'-between columnar and squamous. This is a term that is borrowed from the urinary tract where there is a non-keratinizing multilayered epithelium with specific ultrastructural features and functional attributes. 'Transitional' epithelium in the upper respiratory tract only appears to be so when there is a lack of ultrastructural evidence of its exact nature. If electron microscopical examination were carried out the epithelium would be found to be either a columnar epithelium which has undergone focal stratification or a squamous epithelium with distinct evidence of keratin production.

(3) Melanocytes. Melanin-producing cells are described in the nasal epithelium particularly of the septum and are said to be particularly common in Ugandan natives (Lewis, 1967). Pigmented cells have not been seen in nasal epithelium in the large amount of surgical material which we have examined histologically. Melanin pigmentation of basal layer of squamous epithelium was found to be particularly common in nasopharyngeal specimens of Indians and Malays (Ali, 1965).

(4) Neuroepithelium. The cells of the nasal epithelium receiving olfactory sensation are specialized nerve cells. These structures send a cytoplasmic projection on to the surface where they send out cilia which receive olfactory sensation. The cytoplasm has neurotubules and compressed mitochondria and the deeper aspect of the cell becomes a nerve fibre which links up with the olfactory nerve (Fig. 5).

\section{The neoplams of the nasal epithelium}

Tumours corresponding to each of the above foug types of cell structure can be found arising from the nasal epithelium.

(1) Tumours of pseudostratified columnar epithe® lium (a) Papillary adenoma. A benign neoplasng reproducing the epithelium of the nose has beenvariously known as Schneiderian papilloma, cylin dric cell papilloma and papillary adenoma (Hyams 1971). This lesion grows principally in the maxillarg antrum and lateral wall of the nose. The principas histological feature is an everted frond-like series of. folds of epithelium of ciliated and mucus secretory type. The cilia are often atrophic. Numbers of cystio like mucous cells are present which may be mistakem for rhinosporidiosis (Figs 6 and 7).

(b) Papillary carcinoma. The origin of the papillary adenoma from the nasal epithelium is indicated by its composition of ciliated and mucous cells.

A malignant counterpart would be expected toे have lost the ciliated cells while retaining otheo features. Such a tumour is seen in the papillgry adenocarcinoma. This neoplasm usually presents with nasal obstruction and the nose contains polypoid masses of tumour. Radiological examinat may show bone destruction. Microscopical examinas tion reveals a neoplasm characterized by papillar outgrowths covered by columnar cells showin\% malignant characteristics together with invading malignant glandular formations in which mucus production is active (Figs 8 and 9). These tumours are locally aggressive but have little tendency to metastasize.

Problems arising in connection with the classifices tion of tumours of pseudostratified columnar epithe 3 . lium. The presence of ciliated and mucous cellog together indicates a certain origin of the benign form. of this tumour from nasal epithelium. The malignan form of the lesion may also be of seromucinous glan origin, although the papillary processes invite com? parison with the benign form of the tumour. Con firmation of the specific nature of this tumoug. pattern is found in the observation that papillard adenocarcinoma with mucus secretion is the specific form of neoplasm found to be associated with the occupation of woodworking (Acheson et al., 1968t Cowdell, 1968). Histological classification has thus led to the possibility that prolonged exposure to wood dust may irritate the nasal epithelium t $\Phi$ produce a specific form of neoplasm.

An objective assessment of this malignant conto diton would also have to take into account the possibility in any individual case that this tumoum 


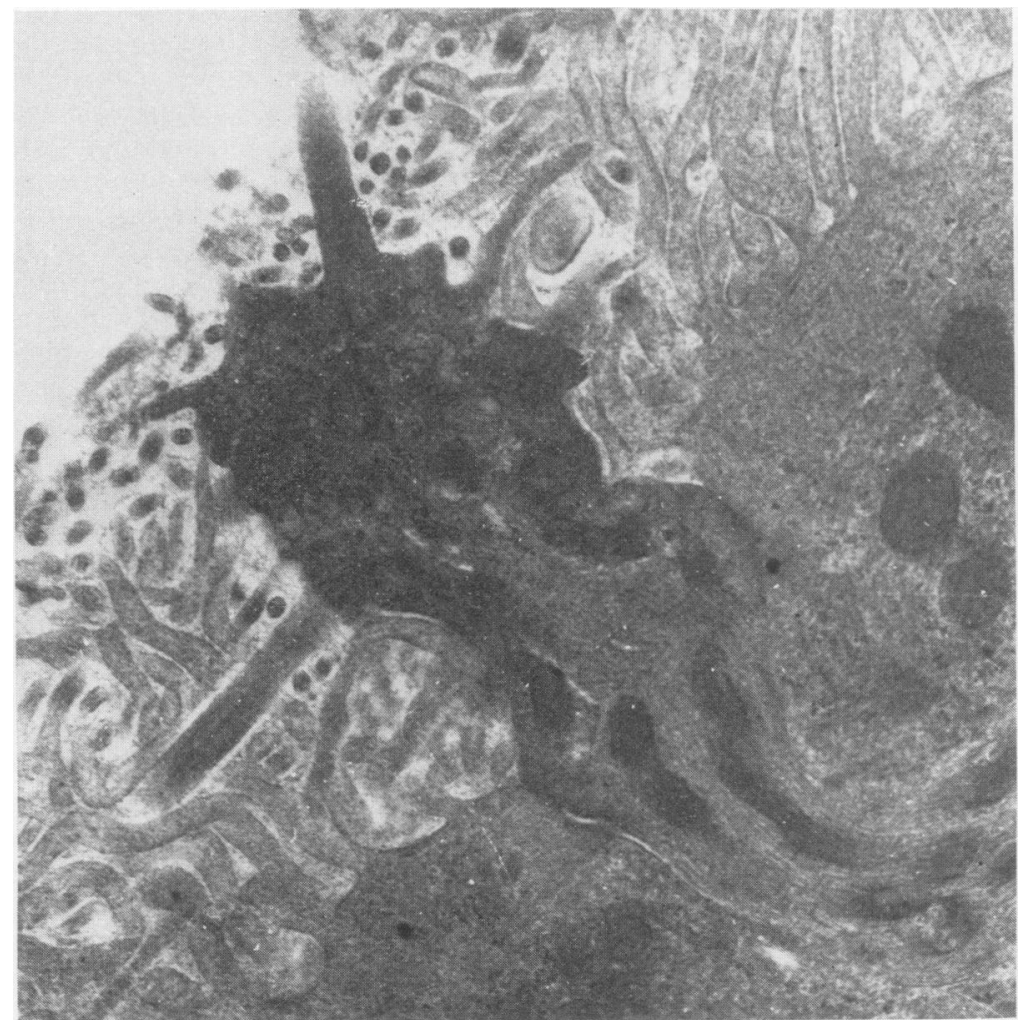

FIG. 5. Olfactory neuroepithelial cell showing ciliated projections and neurotubules in cytoplasm at base of cell. Note microvilli emanating from surfaces of adjacent 'sustentacular' cells. Electron micrograph $\times 25,200$.

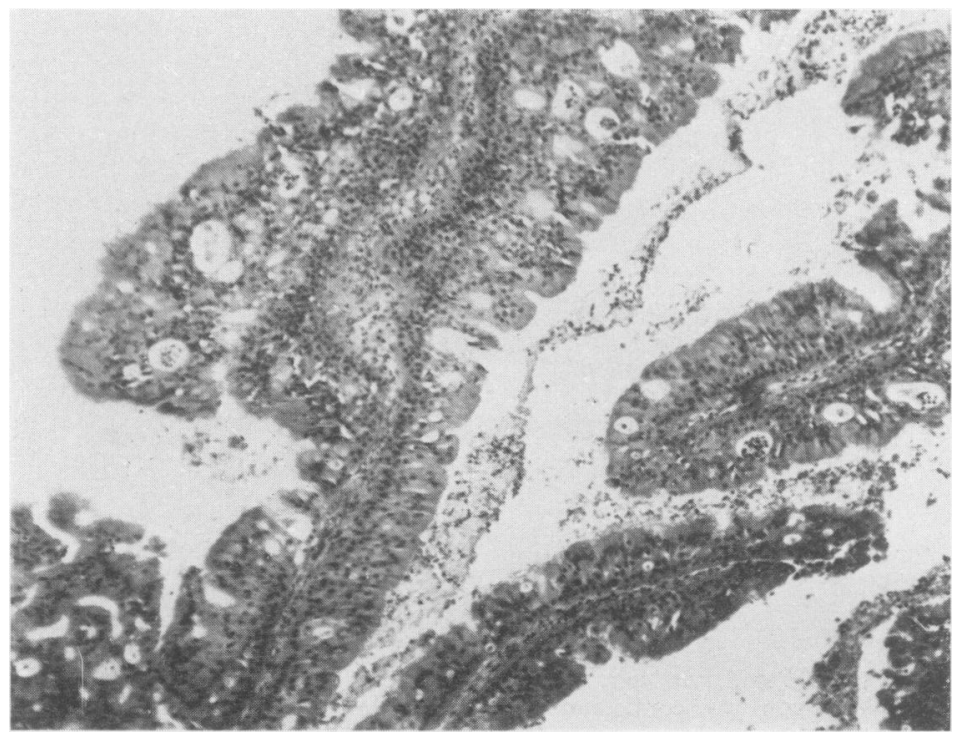

Fig. 6. Papillary adenoma of nasal cavity composed of fronds of mucosa covered by respiratory epithelium. Small cyst-like structures derived from goblet cells are present among the epithelial cells. HE $\times 80$. 


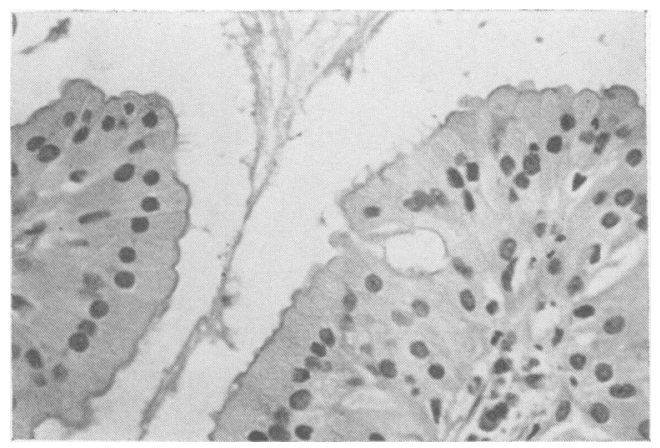

FIG. 7. Papillary adenoma. Note ciliated nature of the columnar cells. The spaces represent atrophic mucus secretory cells. HE $\times 280$.

may be metastatic from a primary in such sites as the ovary, intestine or thyroid. Such a possibility would have to be excluded by clinical investigation.

(2) Tumours of squamous epithelium. (a) Everted or fungiform squamous papilloma. These lesions which are similar to those frequently seen in the uvula or tonsil when arising in the nose are always found in the nasal septum (Hyams, 1971). They are exophytic growths with connective tissue cores in the fronds covered by squamous epithelium. Relics of the columnar epithelium of the nose are often seen in the form of mucous cyst-like structures representing swollen goblet cells. There is no tendency to malignancy in this type of growth.

(b) Inverted papilloma. This tumour arises from the lateral wall of the nose and nasal sinuses, but never from the nasal septum (Hyams, 1971). The neoplasm presents as polypoid masses in the nasal ${ }_{C}$ cavity and sinuses. It may have a similar gross appearance to allergic nasal polyps, but usually is more opaque and never shows an elongated stalk. The microscopic appearance is unlike that seen in흐 any other tumour of the respiratory tract. The surface $\frac{\bar{\omega}}{\sigma}$ of the tumour is inverted into sinus-like tracts which ${ }_{\Omega}^{\mathbb{\Phi}}$ are lined by thick layers of epidermoid cells showingo prickle cell formation and sometimes keratinization.? In places the squamous lining gives way to pseudo- $-\overrightarrow{0}$ stratified columnar ciliated or secretory epithelium $\overrightarrow{\vec{\omega}}$ (Figs 10 and 11). In some places the ciliated cells $\omega$ appear to form a row lining the inner surface of and epidermoid area. Serial sections suggest that this 3 . appearance may be due to abrupt transitions between'? stratified squamous and pseudostratified columnar $\vec{\sigma}$ epithelium, the cells of the latter sending their longo threads of cytoplasm down to the basement mem-is brane at the sides of the former areas. The surfacer of the neoplasm is covered by similar alternating? areas of squamous and columnar epithelium. Thestroma of the tumour commonly shows acute and $O$ chronic inflammation and neutrophils are frequentlyơ seen in considerable numbers infiltrating between the squamous epithelial cells. Eosinophils are commen also in the stroma (Fig. 12).

Electron microscopical examination of eight casts. of inverted papilloma has confirmed the squamotis nature of the heaped-up cells even in areas wheres prickle cell formation and keratinization cannot be detected by the light microscope (Fig. 13).

This lesion may therefore be summarized as $a \frac{\Phi}{\circ}$

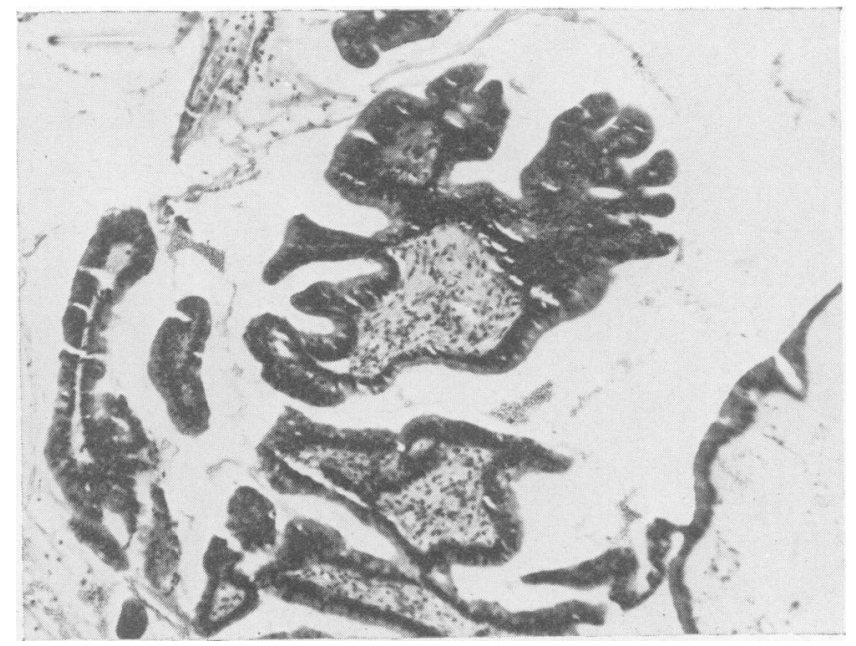

FIG. 8. Papillary adenocarcinoma of nasal cavity. Folds of hyperplastic columnar epithelium are embedded in pools of mucus. The patient was a 72-year-old teacher of carpentry and had been a woodworker all his life. $\mathrm{HE} \times 70$. 


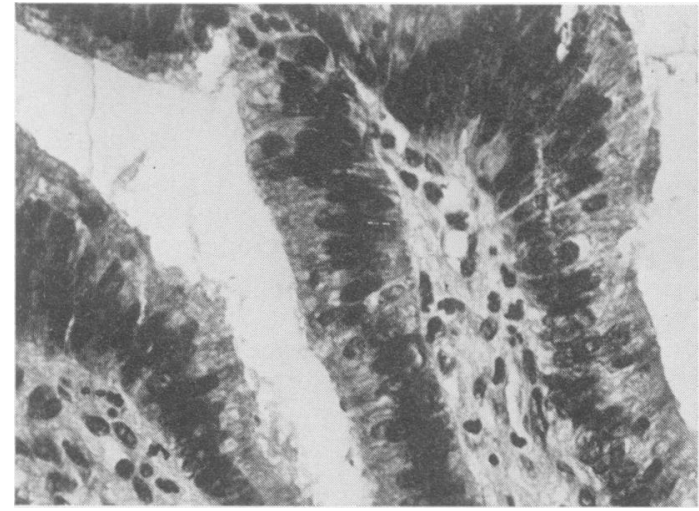

Fig. 9. High powered view of preceding figure. Note columnar cells of active appearance without cilia. $\mathrm{HE} \times 320$.

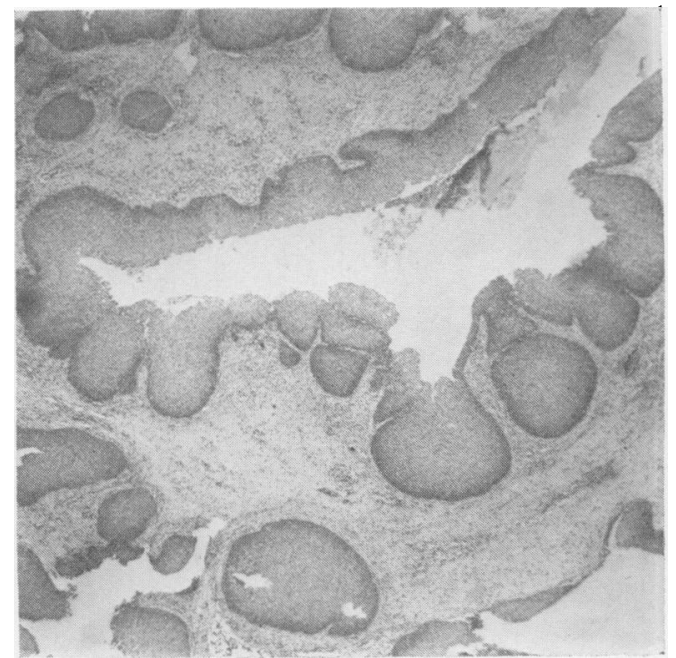

FIG. 10. Inverted papilloma of nose. Sinus-like inversions of the surface epithelium are lined by metaplastic squamous epithelium and an occasional residual focus of respiratory epithelium. HE $\times 17 \cdot 5$.

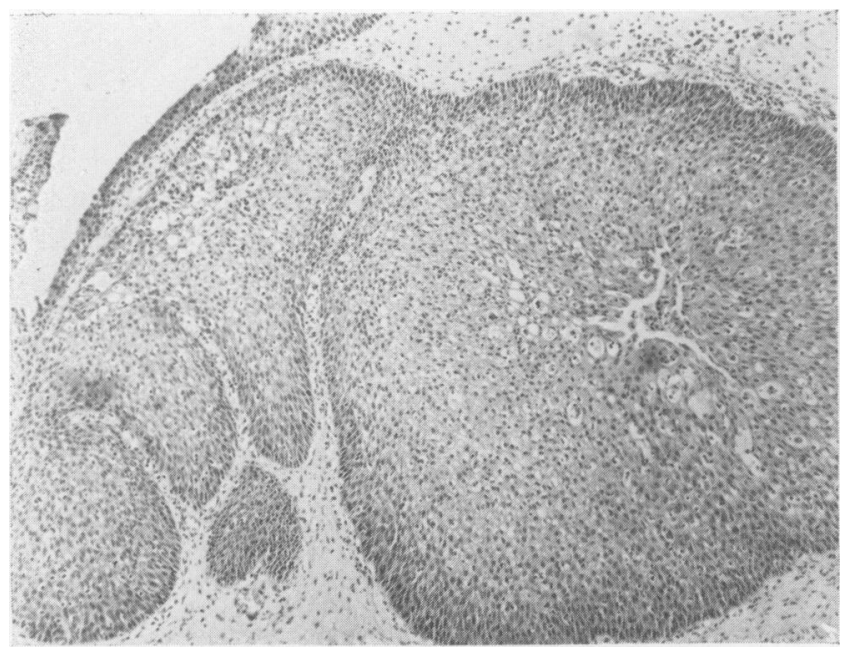

FIG. 11. Squamous lining of sinus-like spaces in inverted papilloma of nose. HE $\times 70$.

widespread and patchy process of squamous metaplasia affecting surface and inverted sinuses of nasal mucosa with concomitant desmoplasia and acute, chronic and allergic types of inflammation. The term 'transitional cell papilloma' is often used to designate this neoplasm. This term is unsatisfactory since the large epithelial formations of the neoplasm are, by both light and electron microscope, undoubtedly squamous and also the most significant feature of the neoplasm is not suggested by that term, namely the sinus formation. Knowledge of these inverted sinuses would be crucial to an understanding of the histogenesis of this common and remarkable lesion. Are they derived from the ducts of seromucinous glands? This is unlikely because the acini of the glands are never seen at the tips of the inverted squamous-lined tracts. The answer lies perhaps in the specific localization of this tumour to the lateral wall of the nose and sinuses in all cases. It is never seen elsewhere although a somewhat 
similar, but not identical, lesion has been described in the urinary bladder (Cummings, 1974). It is conceivable that some feature of the lamina propria in the lateral wall of the nose (e.g. its vascular and

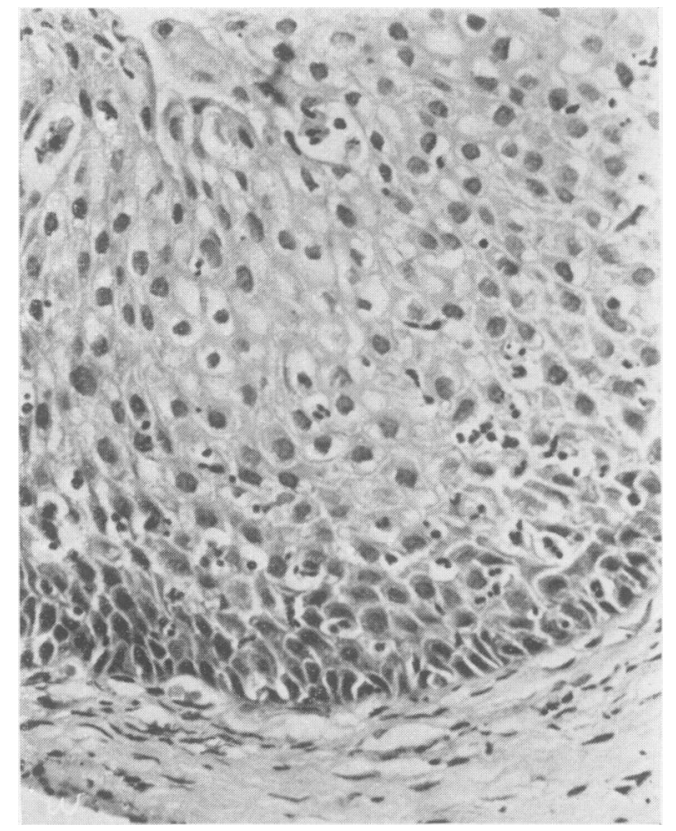

FIG. 12. Neutrophils in squamous epithelium in inverted papilloma of the nose. $\mathrm{HE} \times 280$. loose nature) may cause the development of inverted sinuses when squamous metaplasia arises in the surface epithelium.

There is a strong tendency to recurrence of this tumour. A small but definite relationship to malig nancy exists and ranges from about $5 \%$ (Osborn 1970 ) to $13 \%$ (Hyams, 1971). A figure as low as $2 \%$ is obtained when those cases presenting with simultaneous inverted papilloma and squamou» carcinoma are discounted (Osborn, 1970). ThE malignancy is always of the squamous carcinomatous variety, but may be poorly differentiated.

(c) Squamous carcinoma. These tumours arising in the nasal cavity, nasal sinuses and nasopharyrfe present, when showing evidence of differentiationt. few problems of classification. Tumours are frequently seen in the nose and particularly in the nasos pharynx which, although clearly of epithelial origif show no differentiation either in the squamous direction or towards a glandular structure. Problems of terminology have arisen around these tumours which have appeared in many classifications and have haunted the pathology, surgery and radios therapy of this field for at least 66 years. These problems would seem to arise from three mai sources.

(i) The epithelium of the nasopharynx and alsexof other parts of Waldeyer's ring has a close morpaio logical relationship to the underlying lymphơi tissue (Fig. 14), and indeed recent immunologicat work has shown that there is a shared functio $\bar{B}$ between lymphoid cells and epithelial cells in the

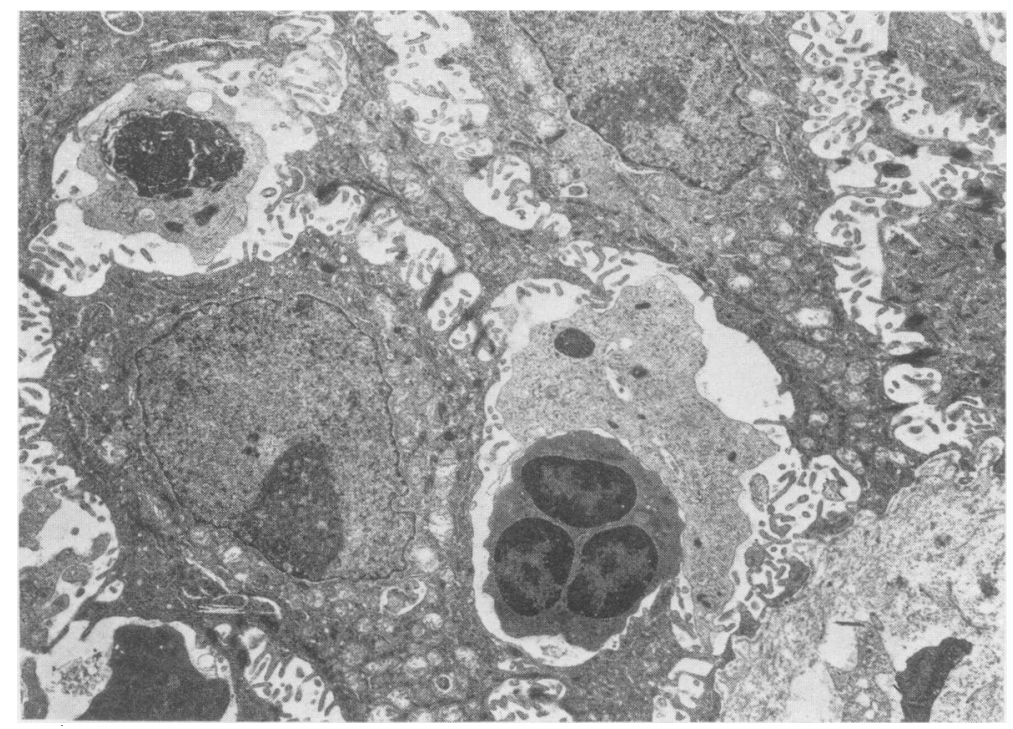

FIG. 13. Inverted papilloma of nose showing desmosomes and tonofibrils in cytoplasm. A neutrophil is seen at bottom centre. Electron micrograph $\times 3600$. 


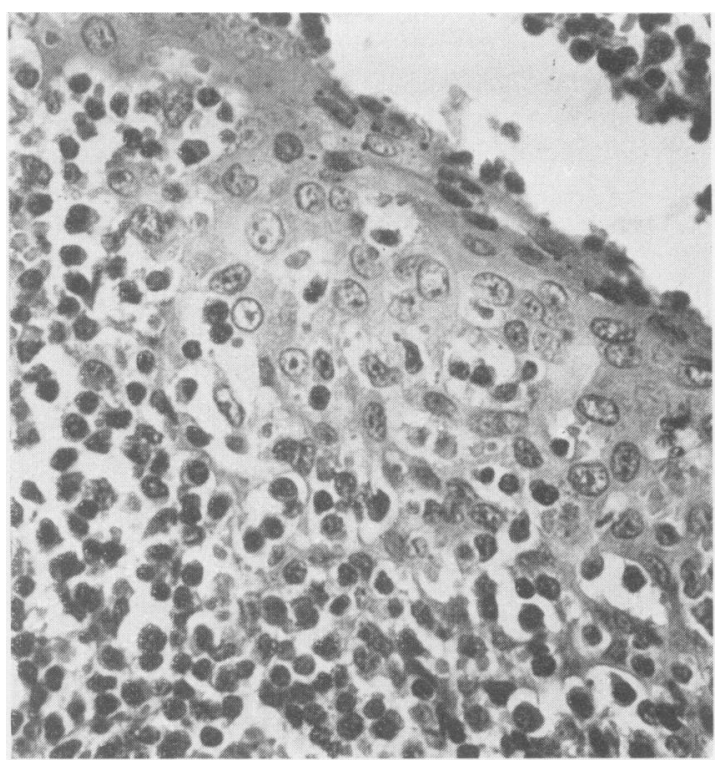

FIG. 14. Lining of normal tonsillar crypt. Above and to the right is a squamous epithelial covering the cells of which mix on its deeper aspect with underlying plasma cells and lymphocytes. HE $\times 400$.

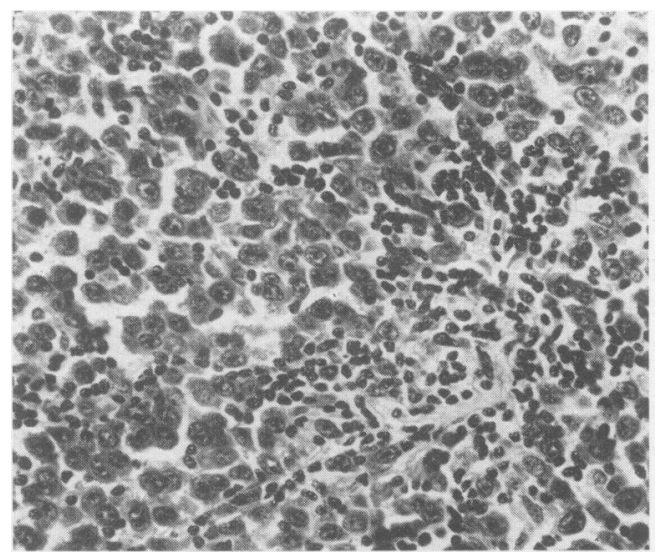

Fig. 15. Undifferentiated carcinoma of nasopharynx. Tumour cells and lymphocytes are closely intermixed giving rise to the term 'lymphoepithelioma' which has commonly been used for these neoplams. HE $\times 240$.

production of IgA. Perhaps largely because of the local presence of large amounts of lymphoid tissue, and also through a continuance of the same relationship in the process of epithelial neoplasia, carcinomas of the nasopharynx are frequently associated with large numbers of lymphocytes (Fig. 15). This has given rise to the term 'lympho-epithelioma', indicating a special form of tumour in which both types of

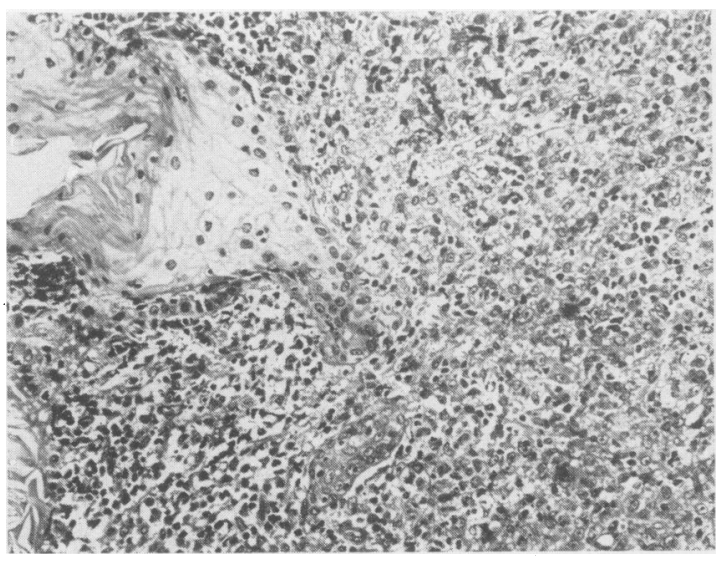

FIG. 16. Anaplastic carcinoma of nasopharynx. Most of the tumour is composed of undifferentiated tumour cells closely associated with lymphocytes. Necrosis is prominent. At the upper left is a squamous epithelial covering the deeper cells of which are conspicuously giving rise to tumour. $\mathrm{HE} \times 150$.

cells share in the neoplastic process. Historically, lympho-epitheliomas have been sub-divided into the Regaud type (Jovin cited by Cappell, 1934) in whiche columns of carcinoma cells are surrounded by lymphocytes, and the Schmincke type (Schmincke cited by Cappell, 1934) in which the carcinoma cells are infiltrated by lymphocytes and may be individually scattered in a sea of lymphocytes.

A study of sections from cases of nasopharyngeal carcinoma has convinced us that these tumours in their undifferentiated form have a similar cellular constitution to more differentiated squamous carcinomas. The latter in some areas have a tendency to lose their prickles and keratinization and the cells in these areas may appear to fuse giving rise to the syncytial effect commonly associated with 'lymphoepithelioma'. The dropping off of tumour cells to form a sarcoma-like neoplasm is well known in squamous carcinomas, particularly in the upper respiratory tract and should such a process take place into a lymphoid stroma, the appearances of lymphoepithelioma will be accounted for. We have found squamous carcinoma in situ or evidence of origin of tumour cells from an overlying squamous epithelium in eleven out of twenty-nine biopsy specimens of nasopharyngeal carcinoma of undifferentiated type (Fig. 16). Electron microscopical examination of these neoplasms, moreover, has revealed, in two cases which we have examined, the abundant tonofibrils and frequent desmosome-bearing intercellular processes characteristic of squamous carcinoma. When these tumours metastasize to a non-lymphoid organ such as the liver they are revealed as strictly 
epithelial tumours, free of lymphocytes. Lymphoepitheliomas are thus poorly differentiated squamous carcinomas. The close relationship of the tumour cells to lymphocytes is possibly more than can be explained on the basis of mere topographical proximity or immunological $\mathrm{T}$ cell reaction, but does not invalidate the essentially squamous cell origin of the neoplasm.

(ii) Tumours of undifferentiated epithelial cells without the lymphoid stroma of lympho-epithelioma have been called transitional cell carcinoma, particularly when forming festoons of tumour cells in continuity with the surface (Ewing cited by Cappell, 1934). Again it is difficult on careful examination of a group of such neoplasms to separate them from squamous carcinoma. Frequently, careful light microscopical examination reveals the hallmarks of keratinizing process. We have, moreover, found evidence of keratin synthesis in each of four tumours, labelled as transitional cell carcinoma by other pathologists, that we have subjected to electron microscopy. It is reasonable to expect that squamous tumours in the upper respiratory tract will lack the markedly keratinizing properties of squamous carcinoma of the skin, just as the mucous membrane squamous epithelium is diminished in this respect.

Because separation of transitional cell carcinomas as a histological entity is difficult, the assertion that they have a better prognosis than other carcinomas (Osborn, 1970) is open to criticism. It seems likely that in putting these tumours into a separate group the proponents of the transitional cell concept have tended to select out two features: (a) those neoplasms which are less keratinized and therefore more radiosensitive than obviously squamous tumours and (b) neoplasms which have fewer features of differentiation than anaplastic carcinomas and, therefore, are biologically less aggressive. A combination of these two features will give rise to a group with better prognosis, but the same results could probably be attained by histological assessment of differentiation by one or other of the standard methods (Michaels, 1974).

(iii) A third source of difficulty in the classification of nasal carcinomas, notably those in the nasopharynx, lies in the badly traumatized appearance that many of the biopsies obtained from this situation show. This is because of the difficulty that is often experienced by the surgeon in taking a satisfactory sample of tissue. Working usually through the mouth without an adequate view of the area to be biopsied, a biopsy from behind and above the soft palate is difficult to obtain without traumatizing the specimen. As a consequence, and without criticism of the surgeon in this procedure, biopsy material from the nasopharynx often shows large areas of artefactual cell damage (Fig. 16). Fre- quently it is only just possible to recognize that there $\stackrel{\mathbb{Q}}{\varrho}$ is a malignant tumour; the biopsy is too damaged. for exact classification. Moreover, since the standard $\Rightarrow$ ? treatment of a nasopharyngeal carcinoma is irradia-? tion, a larger, more satisfactory surgical specimen? will not be forthcoming to remedy the defects in histological identification. It must be admitted that? objective study of nasopharyngeal neoplasms is@ difficult for this reason and it is not surprising thatis confusion exists in the literature on this subject.

(3) Tumours of melanocytes. Benign tumours of melanocytes are extremely rare in the nasal cavity in $\vec{\omega}$ contrast to those arising from the skin of the vesti bular orifice.

TABLE 1. Sites of presentation in sixteen cases of malignant melanoma of nasal passages

\begin{tabular}{|c|c|}
\hline Site & $\begin{array}{l}\text { No. of } \\
\text { cases }\end{array}$ \\
\hline $\begin{array}{cl}\text { Nasal cavity (involving also: } \\
\text { maxillary antrum } & 2 \\
\text { nasal septum } & 3 \\
\text { middle turbinate } & 1 \text { ) }\end{array}$ & 11 \\
\hline Ethmoid sinuses & 1 \\
\hline $\begin{array}{l}\text { Nasopharynx } \\
\text { Nasal vestibule }\end{array}$ & $\begin{array}{l}2 \\
2\end{array}$ \\
\hline
\end{tabular}

Malignant melanomas of the nose in the majox of cases are found in the nasal cavity and may volve the maxillary and ethmoid sinuses and the nasopharynx (Table 1). The tumour may be poly-⿳亠丷厂 poid but it usually presents infiltrating featureš with evidence of destruction of the bony antral wall $\stackrel{\circ}{2}$ A brownish colouration of the tumour is apparen $\overrightarrow{5}$ in approximately two thirds of the cases at operation 3 (Holdcraft and Gallagher, 1969). As elsewhere malignant melanomas of the nose can present aș. tumours composed of cuboidal cells, spindle cells oro a mixture of both. The cellular type is not related to 3 . the degree of malignancy. We believe the identifica cation of melanin pigment in the tumour cells to be essential in the objective light microscopic diagnosiso of malignant melanoma of the nose. The pigment should be shown to be negative for haemosidering and positive by a special staining procedure such ass Fontana's silver stain followed by bleaching with. $20 \mathrm{~V}$ hydrogen peroxide solution. When pigment is absent only electron microscopy can allow a categorical diagnosis of malignant melanoma to be made by the presence of striated precursors of melanin pigment in the cytoplasm of the tumoue cells (Fig. 17). Mitotic figures are unusually frequeno in nasal melanomas, but not of diagnostic importळ The presence of large eosinophilic nuclei has been? stressed as a diagnostic feature of malignant mela $=\frac{0}{T}$ noma of the nose, but this feature was prominent in only $50 \%$ in a series of fourteen cases of malignane 


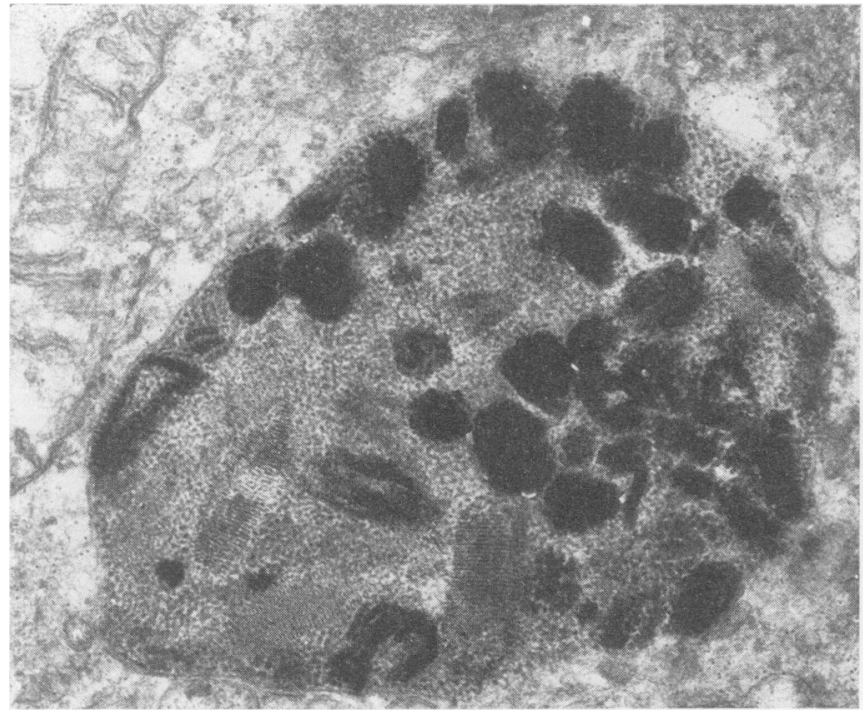

FIG. 17. Crystalline melanin precursor in cytoplasm of nonpigmented malignant melanoma of nasal cavity. Electron micrograph $\times 45,000$.

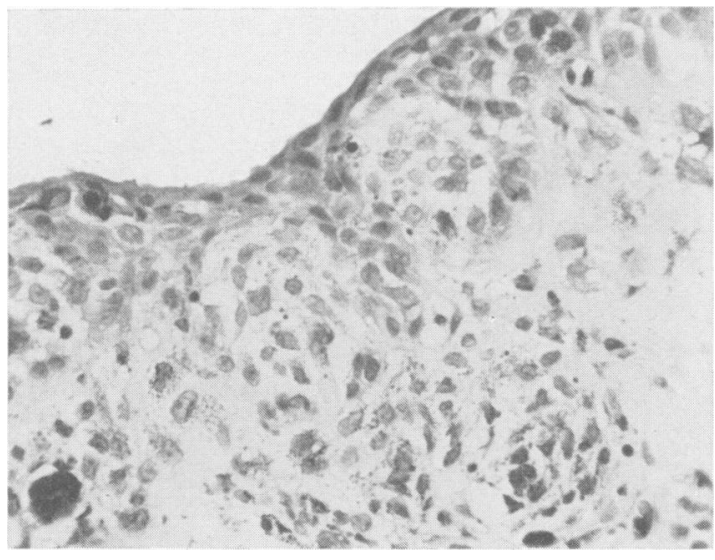

FIG. 18. Area of pigmented junctional activity in epithelium of nose adjacent to a malignant melanoma. $\mathrm{HE} \times 168$.

melanoma of the nose which we examined. Junctional activity is an important feature of dermal melanomas, but this change is unusual in malignant melanoma of the nose, because, unlike the skin tumour, only small amounts of normal nasal mucosa are removed with the tumour. The occasional observation of such changes as well as the clinical pattern of tumour progression in these cases confirms that the neoplasm is indeed of local nasal origin and not, as has been suggested, metastatic from a small skin melanoma (Fig. 18).
(4) Olfactory neuroblastoma. A tumour of the $\vec{\varphi}$ olfactory neurocytes has been more clearly catee ov gorized in recent years. It is also termed olfactor $\bar{\Phi}$ aesthesioneuroblastoma and a more differentiate form, the olfactory aesthesioneurocytoma has also been separated off. Since the normal olfactory neuroepithelium is a differentiated cell the tumour would be expected to be composed of primitive neuroblasts.

The neoplasm originates high up in the nasal cavity; sometimes the ethmoid sinuses are involved and occasionally the maxilla. Occasionally the tumour is found in the nasopharynx (Table 2). Grossly the lesion is smooth, covered by mucosa and frequently polypoid.

TABLE 2. Sites of presentation in twenty-seven cases of olfactory neuroblastoma

\begin{tabular}{lr}
\hline Nasal cavity & 21 \\
Ethmoid sinuses & 5 \\
Cribriform plate region & 2 \\
Maxillary sinus & 2 \\
\hline
\end{tabular}

In two cases more than one site was given.

A characteristic light microscopic pattern has been detected.

The tumour cells, composed of uniform round hyperchromatic nuclei with little cytoplasm, form lobular, well defined masses. The group of cells at the edge of the tumour may be large or small but are 


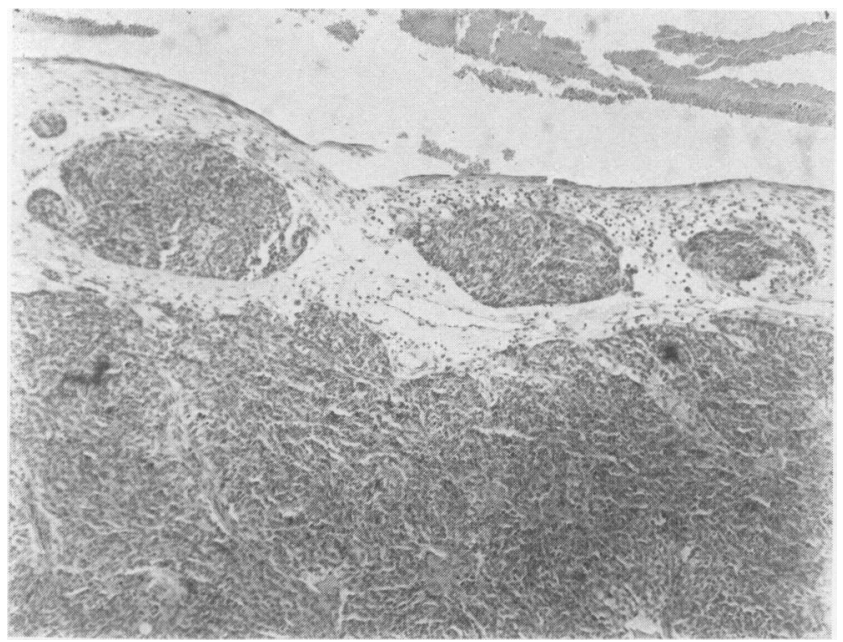

FIG. 19. Olfactoryneuroblastoma. Discrete lobules of tumour are seen beneath nasal epithelium. HE $\times 70$.

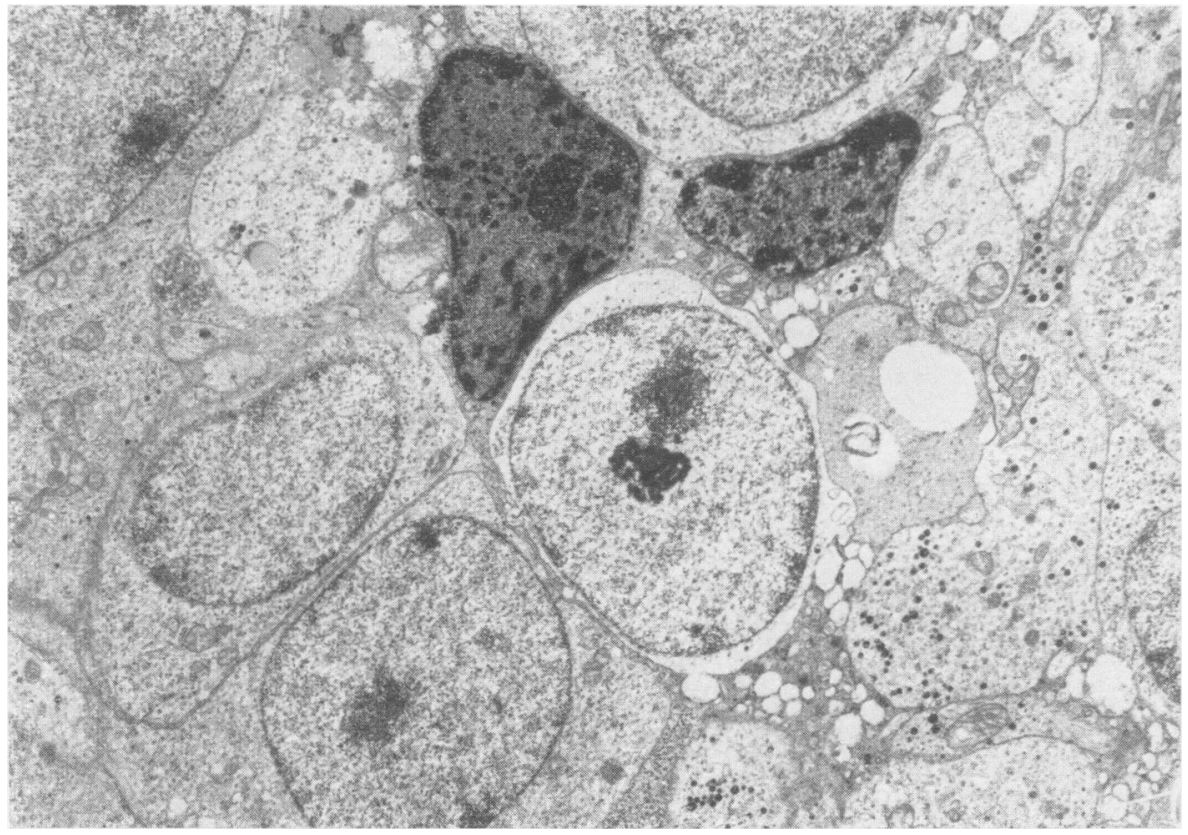

Fig. 21. Olfactory neuroblastoma. To the right of the illustration, in particular, strands of cytoplasm contain electron dense round bodies. Electron micrograph $\times 4200$. 


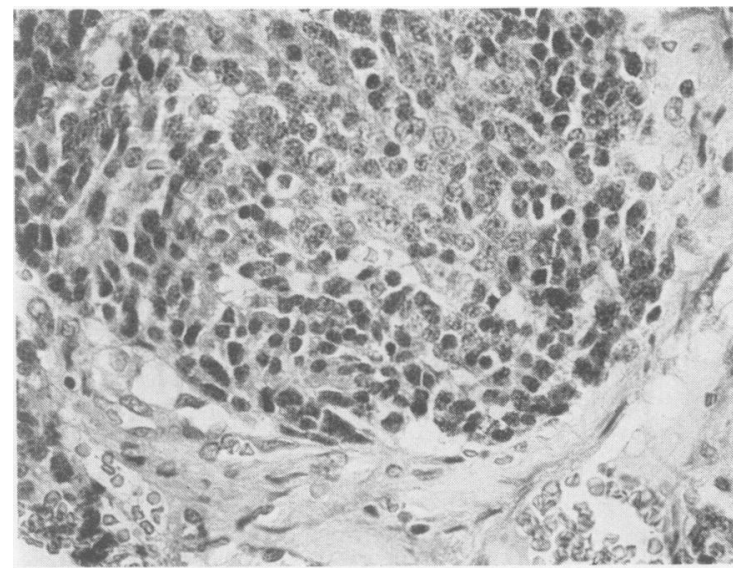

FIG. 20. Olfactory neuroblastoma. Tumour is composed of small darkly staining neuroblasts. $\mathrm{HE} \times 320$.

almost always rounded off into discrete collections and rarely appear to trail off as individual cells (Figs 19 and 20). Rounded spaces are often seen among tumour cells and represent 'rosettes' of the 'pseudo' variety. Flexner-Wintersteiner (glandular type) rosettes and Homer-Wright (enclosing fibrillary material) rosettes are rarely seen. The stroma of the tumour shows a marked vascularity. An axon stain such as the Bodian stain shows occasional fine axis cylinders between tumour cells in a few cases.

The above light microscopic features are distinctive even when the axon stain is negative and there are no rosettes, which is in the majority of cases. Electron microscopical examination has been carried out in a few cases. It reveals membrane-bound neuro-secretory granules in the cytoplasm of the tumour cells and, in some cases, neurites with neurofilaments and neurotubules (Kahn, 1974) (Fig. 21).

The olfactory neuroblastoma is locally aggressive and a small number of cases also develop cervical lymph node and distant metastases.

\section{References}

Acheson, E.O., Cowdell, R.H., Hadfield, E.H. \& MaCBETH, R.G. (1968) Nasal cancer in woodworkers in the furniture industry. British Medical Journal, 1, 587.

Alı, M.Y. (1965) Histology of the human nasopharyngeal mucosa. Journal of Anatomy, 99, 657.

Cappele, D.F. (1934) On lymphoepithelioma of the nasopharynx and tonsils. Journal of Pathology and Bacterio$\log y, 34,49$.

CoWDELl, R.H. (1968) Personal communication.

Cummings, R. (1974) Inverted papilloma of the bladder. Journal of Pathology, 112, 225.

Gould, V.E., WENK, R. \& Sommers, S.C. (1971) Ultrastructural observations on bronchial epithelial hyperplasia and squamous metaplasia. Cancer, 28, 426.

HoldCraft, J. \& Gallagher, J.C. (1969) Malignant melanomas of the nasal and paranasal sinus mucosa. Annals of Otology, Rhinology and Laryngology, 78, 5.

Hyams, V.J. (1971) Papillomas of the nasal cavity and paranasal sinuses. A clinicopathological study of 315 caseso Annals of Otology, Rhinology and Laryngology, 80, 192.

KAHN, L.B. (1974) Esthesioneuroblastoma: a light an electron microscopic study. Human Pathology, 5, 364.

LEWIS, M.G. (1967) Malignant melanoma of the nasal cavity in Ugandan Africans. Cancer, 20, 1699.

MichaELS, L. (1975) Differentiation of squamous carcinoma of the larynx. Canadian Journal of Otolaryngology (in press).

OSBORN, D.A. (1970) Nature and behaviour of transitional tumours in the upper respiratory tract. Cancer, 25, 385. 